\title{
Cell-to-Cell Movement of Potato virus X: The Role of p12 and p8 Encoded by the Second and Third Open Reading Frames of the Triple Gene Block
}

\author{
Atsushi Tamai and Tetsuo Meshi \\ Department of Botany, Graduate School of Science, Kyoto University, Sakyo-ku, Kyoto 606-8502, Japan \\ Submitted 3 April 2001; Accepted 12 June 2001.
}

\begin{abstract}
Potato virus $X(\mathrm{PVX})$ requires three proteins, p25, p12, and $\mathrm{p8}$, encoded by the triple gene block plus the coat protein (CP) for cell-to-cell movement. When each of these proteins was co-expressed with a cytosolic green fluorescent protein (GFP) in the epidermal cells of Nicotiana benthamiana by the microprojectile bombardment-mediated gene delivery method, only p12 enhanced diffusion of coexpressed GFP, indicating an ability to alter plasmodesmal permeability. p25, p12, and CP, expressed transiently in the initially infected cells, transcomplemented the corresponding movement-defective mutants to spread through two or more cell boundaries. Thus, these proteins probably move from cell to cell with the genomic RNA. In contrast, p8 only functioned intracellularly and was not absolutely required for cell-to-cell movement. Since overexpression of p12 overcame the $\mathrm{p8}$ deficiency, $\mathrm{p8}$ appears to facilitate the functioning of p12, presumably by mediating its intracellular trafficking. Considering the likelihood that p12 and $\mathrm{p8}$ are membrane proteins, it is suggested that intercellular as well as intracellular movement of PVX involves a membrane-mediated process.
\end{abstract}

It is well accepted that most plant viruses move from cell to cell via plasmodesmata, which are plasma membrane-lined intercellular connections consisting of a cytoplasmic annulus and a desmotubule, a central appressed form of the endoplasmic reticulum (ER) (Lucas et al. 1993; Overall and Blackman 1996; Van Bel and Van Kesteren 1999). Many viruses encode one or more movement proteins (MPs) that facilitate transport of viral nucleic acids through plasmodesmata into neighboring cells (Atabekov and Taliansky 1990; Deom et al. 1992; Maule 1991). It has been shown that various MPs target to plasmodesmata, modify the plasmodesmal size exclusion limit (SEL), bind to RNAs, and in some instances, associate with the ER and cytoskeletons (Carrington et al. 1996; Lazarowitz and Beachy 1999). The molecular mechanisms by which replicated viral genomes move from the site of replication to plasmodesmata and, subsequently, through them, however, are still largely unknown.

A number of virus groups, including potexviruses, hordeiviruses, benyviruses, and pecluviruses, encode three proteins involved in their cell-to-cell movement (Beck et al.

Corresponding author: T. Meshi; Fax: +8175753 4141;

E-mail: tmeshi@gr.bot.kyoto-u.ac.jp
1991; Gilmer et al. 1992; Herzog et al. 1998; Petty and Jackson 1990), which are translated from partially overlapping open reading frames (ORFs), termed the triple gene block (TGB) (Morozov et al. 1989). These three proteins are generically referred to as TGBp1, TGBp2, and TGBp3, in order of the ORFs (Solovyev et al. 1996). In the case of Potato virus $X$ $(\mathrm{PVX})$ and other potexviruses, the coat protein $(\mathrm{CP})$ also is required for cell-to-cell movement, and all four proteins are dispensable for replication (Baulcombe 1995; Beck et al. 1991; Chapman et al. 1992; Forster et al. 1992).

TGBp1 shares a domain with a similarity to RNA helicases (Gorbalenya and Koonin 1989; Morozov et al. 1989; Wong et al. 1998) and has ATPase and RNA binding activities (Bleykasten et al. 1996; Donald et al. 1997; Kalinina et al. 1996; Liou et al. 2000; Morozov et al. 1999; Rouleau et al. 1994; Wung et al. 1999). Microinjection studies indicate that the TGBp1 proteins of PVX and White clover mosaic virus (WClMV, a potexvirus) are able to increase the SEL of plasmodesmata and move through them (Lough et al. 1998). Biolistic studies confirmed that a PVX TGBp1-green fluorescent protein (GFP) fusion can facilitate its own cell-to-cell movement (Yang et al. 2000). Potexvirus TGBp1, however, has been found predominantly in cytoplasmic laminate inclusions and has not been localized in plasmodesmata of infected cells (Chang et al. 1997; Davies et al. 1993; Rouleau et al. 1994). Angell and colleagues (1996) showed that cell-to-cell movement of PVX is associated with a plasmodesmal modification, resulting in an increase in SEL. For this plasmodesmal modification, expression of TGBp1 is required but not solely responsible (Angell et al. 1996).

TGBp2 is most conserved among the TGB-encoded proteins, whereas TGBp3 has diverged considerably and falls into several groups with no significant similarity (Solovyev et al. 1996). TGBp2 and TGBp3 contain domains rich in hydrophobic amino acids, a typical feature of integral membrane proteins (Morozov et al. 1989). These proteins associate with membrane and cell wall fractions in vitro and in vivo (Donald et al. 1993; Hefferon et al. 1997; Morozov et al. 1990; Morozov et al. 1991; Niesbach-Klösgen et al. 1990). Lough and colleagues (2000) suggested, on the basis of the data from transcomplementation experiments, that TGBp2 and TGBp3 only functions intracellularly in potexvirus cell-to-cell movement.

Electron microscopy studies revealed that PVX CP and virion-like filamentous particles accumulate in plasmodesmata upon infection (Oparka et al. 1996; Rouleau et al. 1995, Santa Cruz et al. 1998). CP alone, however, does not have the ability 
to target itself to plasmodesmata, mediate its own transport, or increase the plasmodesmal SEL (Lough et al. 1998; Oparka et al. 1996; Santa Cruz et al. 1998). Recently, studies with PVX and WCIMV suggested that the viral ribonucleoprotein complex in which the virus moves from cell to cell may include CP and TGBp1 (Lough et al. 1998).

In this study, we examined the functions of the TGBencoded proteins and the $\mathrm{CP}$ of PVX in regard to the ability to modulate plasmodesmal permeability and to transcomplement movement-defective phenotype by means of microprojectile bombardment. Our work sheds new light on the role of TGBp2 and TGBp3: i) p12 (TGBp2 of PVX), but not p25 (TGBp1), increases plasmodesmal permeability as evaluated by the cell-to-cell trafficking of a co-expressed cytosolic GFP; ii) p12, p25, and $\mathrm{CP}$ can function multicellularly to enable the corresponding mutants to spread through two or more cell boundaries; and iii) p8 (TGBp3) is not absolutely required for
PVX movement and probably facilitates the functioning of p12. In light of these findings, we discuss cell-to-cell movement of PVX.

\section{RESULTS}

\section{Enhanced cell-to-cell trafficking of a cytosolic GFP by co-expression of p12.}

G3GFP is a soluble GFP variant with high fluorescence intensity (Kawakami and Watanabe 1997). When pBIG3 (Fig. 1A), from which G3GFP is expressed under the control of the 35S RNA promoter of Cauliflower mosaic virus, was bombarded into mature leaves of Nicotiana benthamiana, GFP fluorescence was restricted to single, isolated epidermal cells at $94 \%$ of transfected sites at $24 \mathrm{~h}$ postbombardment (Table 1). At the other sites, a small fraction of the expressed G3GFP moved at a detectable level from the bombarded cells to
A

\section{p35X25}

(p25)

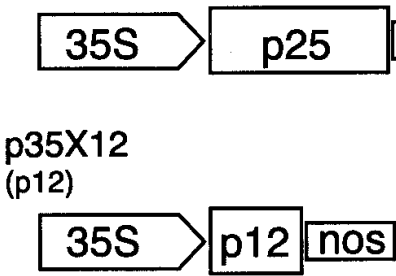

p35X8

(p8)

$35 S$

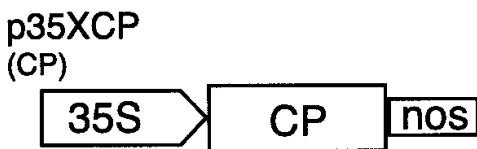

pBIG3

(G3GFP)

35S

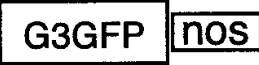

B

piX.erG3

(PVX.erG3)

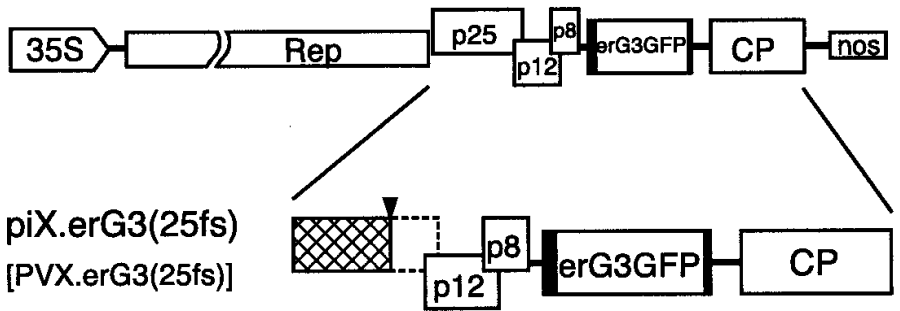

piX.erG3(12fs)

[PVX.erG3(12fs)]

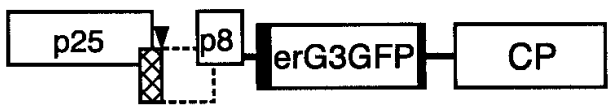

piX.erG3(8dm)

[PVX.erG3(8dm)]

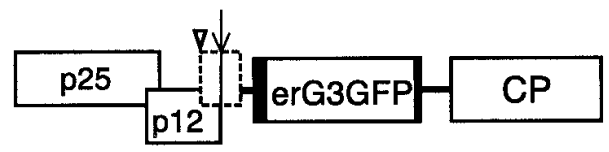

piX.erG3(Cd)

[PVX.erG3(Cd)]
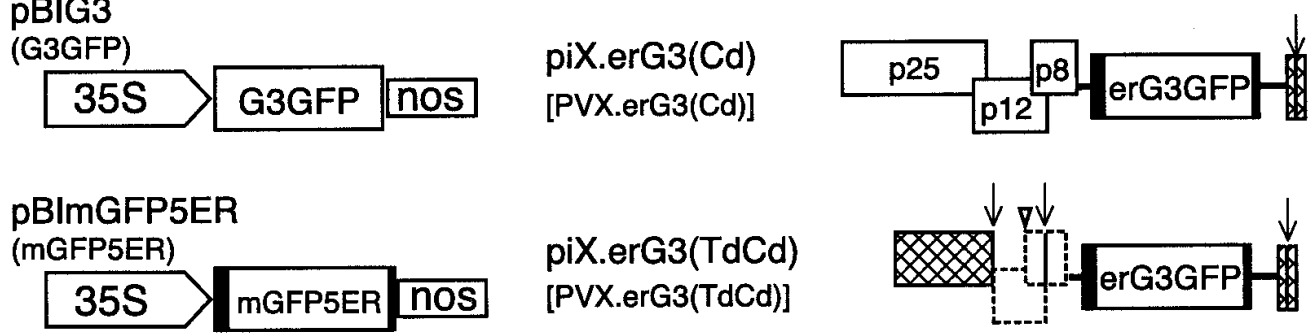

piX.erG3(TdCd) [PVX.erG3(TdCd)]

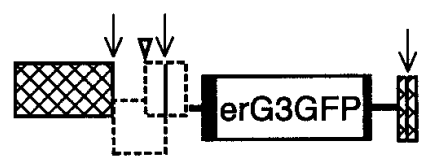

Fig. 1. Constructs used for microprojectile bombardment. A, Schematic representation of the constructs expressing a triple gene block-encoded protein, the coat protein, or a green fluorescent protein derivative. Each gene is expressed under the control of the Cauliflower mosaic virus 35S RNA promoter (35S) and has the nopaline synthase gene terminator. The protein expressed from each plasmid is shown in parentheses below the name of the plasmid. The endoplasmic reticulum localization and retention signals in mGFP5ER are shown with thick, black lines. B, Schematic representation of infectious Potato virus $X$ plasmids. Progeny virus names are indicated below the plasmid names. Coding regions and noncoding regions are indicated with boxes and thin lines, respectively. Closed triangles indicate the frame-shift mutations introduced into the p 25 or p12 open reading frame (ORF). Open triangles indicate the mutated initiation codon of the p8 ORF. Deletions are marked with arrows. Boxes delineated with broken lines indicate nonexpressed portions, and cross-hatched boxes indicate the expressed portions of the truncated proteins. 
neighboring cells, forming halos. Such low frequency of halo formation is characteristic of the source leaves of $N$. benthamiana (Oparka et al. 1999). When a tobamoviral or cucumoviral MP, which is known to increase the plasmodesmal SEL (Ding et al. 1995; Vaquero et al. 1994; Waigmann et al. 1994; Wolf et al. 1989), is co-expressed, the cell-to-cell trafficking of G3GFP is enhanced greatly (Tamai and Meshi 2001) and, consequently, 50 to $60 \%$ of the GFP-positive sites in our assay conditions exhibited halos (Table 1: 30K MP of Tomato mosaic virus [ToMV, a tobamovirus] and 3a MP of Cucumber mosaic virus [CMV, a cucumovirus]). Such an increase in the frequency of halo formation probably reflects the ability of MPs to modulate plasmodesmal permeability (Tamai and Meshi 2001). We used this method to examine the activity of the PVX-encoded proteins involved in cell-to-cell movement.

Figure 2 shows typical results obtained when pBIG3 was co-bombarded with p35X25, p35X12, p35X8, or p35XCP, from which p25, p12, p8, or CP was expressed (Fig. 1A). Only p12 was able to enhance cell-to-cell trafficking of G3GFP (Fig. 2B). The frequency of halo formation mediated by 12 was comparable to that observed when G3GFP was co-expressed with ToMV 30K MP or CMV 3a MP (Table 1). In addition, GFP fluorescence was sometimes detected in cells not adjacent to the highly fluorescent, bombarded cells. Thus, p12 is likely to have plasmodesmal permeabilitymodulating activity. In contrast, neither p25, p8, nor $\mathrm{CP}$ enhanced cell-to-cell trafficking of G3GFP (Fig. 2A, C, and $\mathrm{D}$ and Table 1). The failure to detect such activity for these three proteins was not the result of a lack of their expression from the bombarded plasmids because the same constructs transcomplemented the movement-defective phenotype of PVX mutants with a mutation or mutations in the corresponding genes (see below).

\section{Multicellular functioning of p25, p12, and CP} in transcomplementation of movement-defective PVX.

Infectious PVX clones have been developed from which replication-competent PVX derivatives are generated after microprojectile bombardment into leaves (Baulcombe et al. 1995). When an infectious plasmid producing a movementdefective PVX mutant is co-bombarded with the separately cloned corresponding gene, the mutant spreads from the bombarded cells to neighboring cells (Lough et al. 2000; Morozov et al. 1997). We performed such transcomplementation experiments to clarify the activities of the TGB-

Table 1. Activity of Potato virus X (PVX)-encoded proteins to potentiate cell-to-cell trafficking of cytosolic green fluorescent protein (GFP)

\begin{tabular}{lc}
\hline Expressed protein(s) & Halo formation frequency \\
\hline G3GFP only & $6 / 100(6)$ \\
G3GFP + PVX p25 & $17 / 283(6)$ \\
G3GFP + PVX p12 & $108 / 192(56)$ \\
G3GFP + PVX p8 & $12 / 199(6)$ \\
G3GFP + PVX CP & $10 / 144(7)$ \\
G3GFP + Tomato mosaic virus 30K & $60 / 115(52)$ \\
G3GFP + Cucumber mosaic virus 3a & $30 / 47(64)$ \\
\hline
\end{tabular}

a Data are presented as the number of bombarded sites containing two or more GFP-positive cells/total number of bombarded sites examined at $24 \mathrm{~h}$ postbombardment. Frequency $(\%)$ is shown in parentheses. G3GFP alone was trafficked between trichome cells and therefore the trichome cells were not counted. encoded proteins and CP within the context of viral cell-tocell movement.

We first modified the PVX vector pPVX201 (Baulcombe et al. 1995), so that the ER-localized GFP variant erG3GFP (Tamai and Meshi 2001) was expressed replication-dependently from the duplicated CP subgenomic promoter. Because ER-localized GFP does not traffic between cells (see below) (Oparka et al. 1999; Tamai and Meshi 2001), it is possible to identify infected cells directly by fluorescence microscopy. When the resulting plasmid, piX.erG3 (Fig. 1B), was bombarded into mature leaves of $N$. benthamiana, clusters of GFPexpressing cells were observed (Fig. 3A). This indicates that the virus derived from piX.erG3, named PVX.erG3, was competent in replication and movement. Hereafter, progeny viruses are indicated simply by substituting the prefix PVX for the prefix piX in the name of the progenitor plasmid (Fig. 1B).

p25- and p12-deficient constructs, piX.erG3(25fs) and piX.erG3(12fs), were made by introducing a frame-shift mutation in the respective genes (Fig. 1B). The same or similar mutations have been shown previously to result in a movement-defective phenotype (Lough et al. 2000; Morozov et al. 1997; Verchot et al. 1998). The CP-deficient mutant clone piX.erG3(Cd) had a 636-bp deletion (nucleotides 5,667 to 6,302), lacking most of the CP ORF (Fig. 1B). When these constructs were bombarded alone, GFP fluorescence was restricted completely to single cells (Fig. 3B to D and Table 2), confirming that expression of $\mathrm{p} 25, \mathrm{p} 12$, and $\mathrm{CP}$ is required for cell-to-cell movement.

When these mutant constructs were co-bombarded with a $\mathrm{p} 35 \mathrm{X}$ series plasmid having the corresponding wild-type gene,
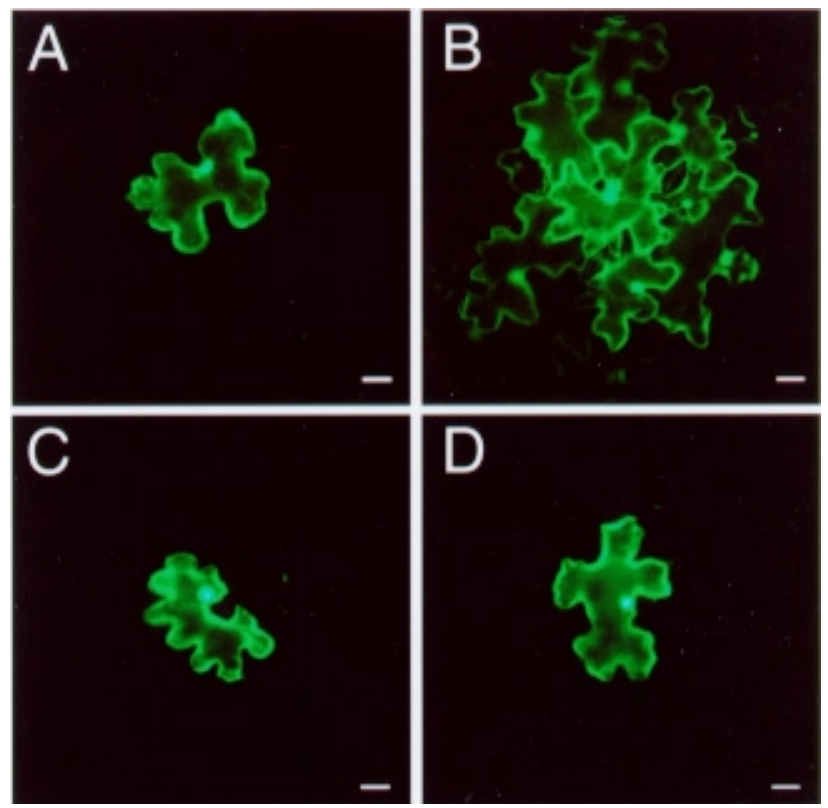

Fig. 2. The ability of Potato virus $X$-encoded proteins to increase plasmodesmal permeability. A, Co-bombardment of pBIG3 expressing a cytosolic green fluorescent protein (G3GFP) together with p35X25 expressing $\mathrm{p} 25$. B, Co-bombardment of pBIG3 with p35X12 expressing p12. C, Co-bombardment of pBIG3 with p35X8 expressing p8. D, Cobombardment of pBIG3 with p35XCP expressing CP. G3GFP fluorescence was restricted to the bombarded cell (A and C-D) or formed a halo (B). Images were taken under blue-light irradiation $24 \mathrm{~h}$ postbombardment. Bars $=25 \mu \mathrm{m}$. 
transcomplementation was observed at a frequency of 90 to 96\% (data not shown), confirming that these proteins can function in trans (Ares et al. 1998; Lough et al. 2000; Morozov et al. 1997; Spillane et al. 1997). The size of infection foci formed by the action of p12, however, appeared larger than that expected if p12 functioned only in the bombarded cells (Lough et al. 2000). We then examined the number of cell boundaries each movement-defective mutant could move from the initially infected (bombarded) cells in the presence of p25, p12, or CP. For this purpose, the inoculum included a third plasmid, pBImGFP5ER (Fig. 1A), expressing the UV-excitable, ER-localized GFP variant mGFP5ER (Haseloff and Siemering 1998). Because virally expressed erG3GFP is not or little, if any, excited by UV light (Tamai and Meshi 2001), the primary infected cells can be found by UV irradiation among clusters of erG3GFPpositive cells that are visible by blue-light irradiation. In transcomplementation experiments, we collected data at 3- day postbombardment because at 2-day postbombardment, GFP fluorescence was still weak; infection foci were close to their maximum size at 3-day postbombardment; and longer incubation should be avoided to minimize the possible generation of revertants and the possible effects of the host resistance response.

PVX.erG3(25fs), PVX.erG3(12fs), and PVX.erG3(Cd) spread from the initially infected cells through two or more cell boundaries in 76 to $83 \%$ of the foci in which complementation occurred (Fig. 4 and Table 3 ). These results clearly demonstrate that the separately cloned $\mathrm{p} 25, \mathrm{p} 12$, and $\mathrm{CP}$ genes exert their effects not only in the initially infected cells but also in their surrounding cells. It is therefore highly likely that all these proteins, including p12, traffic from cell to cell concurrently with the viral genome. It is noted that mGFP5ER did not detectably move out from the bombarded cells, even in the presence of all the PVX-encoded proteins and the replicating virus (Fig. 4).


Fig. 3. Phenotype of green fluorescent protein (GFP)-tagged Potato virus $X$ (PVX) mutants in relation to cell-to-cell movement. A, Bombardment of piX.erG3. The resulting GFP-tagged virus, PVX.erG3, spread multicellularly. B, Bombardment of piX.erG3(25fs) with a frame-shift mutation in the p25 open reading frame (ORF) results in single-cell infection. $\mathbf{C}$, Bombardment of piX.erG3(12fs) with a frame-shift mutation in the p12 ORF results in single-cell infection. D, Bombardment of piX.erG3(Cd) with an internal deletion in the coat protein (CP) ORF results in single-cell infection. $\mathbf{E}$ and $\mathbf{F}$, Bombardment of piX.erG3(8dm), producing the p8-deficient mutant PVX.erG3(8dm). Single-cell infection (E) and infection of two adjoining cells $(\mathbf{F})$. G, Bombardment of piX.erG3(TdCd) defective in all triple gene block proteins and the CP, resulting in single-cell infection. $\mathbf{H}$, Bombardment of piX.erG3(TdCd) together with p35X25, p35X12, and p35XCP. Clustered cells were infected in the absence of p8. I, Bombardment of piX.erG3(TdCd) together with p35X25, p35X12, p35X8, and p35XCP. Clustered cells were infected. J, Co-bombardment of piX.erG3(8dm) and p35X12. Infected cells formed a small cluster. K, Co-bombardment of piX.erG3(25fs) and p35X12, resulting in single-cell infection. L, Co-bombardment of piX.erG3(Cd) and p35X12, resulting in single-cell infection. Images were taken 3 days postbombardment under blue-light irradiation. Bars $=50 \mu \mathrm{m}$. 
Incomplete requirement of $\mathrm{p} 8$ in cell-to-cell movement and restoration of the $\mathrm{p8}$ deficiency by overexpression of $\mathrm{p} 12$.

To inactivate the $\mathrm{p} 8 \mathrm{ORF}$, we first introduced a 43-bp deletion (nucleotides 5,496 to 5,538) into piX.erG3. This mutation concomitantly caused a frame shift in the 24th codon of the p8 $\mathrm{ORF}$, and the resulting protein lacked the C-terminal 47 amino acids of the wild-type p8. This mutation was not enough to confine the resulting virus in isolated cells, however, because approximately $10 \%$ of the infection sites consisted of 2 to 4 GFP-positive cells (data not shown). Because the possibility remained that the leaky phenotype might be the result of expression of the $\mathrm{N}$-terminal portion of $\mathrm{p} 8$, which was derived from the part overlapping with the p12 ORF, an additional mutation was made at the initiation codon of the $\mathrm{p} 8$ ORF by changing AUG into ACG. Note that this U-to-C substitution did not cause the amino acid change in p12, and the same mutation was used previously to inactivate the p8 ORF (Lough et al. 2000). When the resulting plasmid, piX.erG3(8dm) (Fig. 1), was bombarded alone, a small cluster of infected cells were still observed at a frequency of $14 \%$ (Fig. 3E and F and Table 2), just like the initially designed construct. Two adjoining cells were fortuitously bombarded simultaneously at a low frequency. However, no such case was observed for the p25-, p12-, or CP-deficient mutant (Table 2). Thus, the leaky phenotype of PVX.erG3(8dm) indicates that PVX can move from cell to cell even in the absence of p8, although the process is inefficient.

To clarify whether $\mathrm{p} 8$ is really dispensable for PVX to move from cell to cell, we prepared piX.erG3(TdCd), in which all the TGB and the CP ORF were inactivated (Fig. 1). As expected, the bombardment of piX.erG3(TdCd) resulted in singlecell infections (Fig. 3G and Table 2). When piX.erG3(TdCd) was co-bombarded with the plasmids $\mathrm{p} 35 \times 25, \mathrm{p} 35 \times 12$, and p35XCP, 34\% of the infection foci had two or more GFPpositive cells (Fig. $3 \mathrm{H}$ and Table 2). Therefore, PVX.erG3(TdCd), lacking in all TGB-encoded proteins and $\mathrm{CP}$, can move from the initially infected cells to neighboring cells when p25, p12, and CP are supplied in trans. When p35X8 encoding p8 was additionally included in the inoculum
(Fig. 3I), the frequency of transcomplementation increased greatly $(82 \%)$ (Table 2$)$, comparable to that observed for the transcomplementation of PVX.erG3(8dm) with p8 (91\%) (Table 2). These results show that expression of $\mathrm{p} 8$ is not absolutely required for PVX to move from an epidermal cell of $N$. benthamiana to its adjoining cells but is necessary for efficient movement.

Interestingly, the frequency of cell-to-cell movement of PVX.erG3(TdCd) in the presence of p25, p12, and CP was higher than that observed for the p8-deficient mutant PVX.erG3(8dm) (34\% versus 14\%) (Table 2). This might result from overexpressing a protein from a bombarded plasmid. To examine this possibility, piX.erG3(8dm) was bombarded with $\mathrm{p} 35 \mathrm{X} 25, \mathrm{p} 35 \mathrm{X} 12$, or $\mathrm{p} 35 \mathrm{XCP}$. Surprisingly, as many as $60 \%$ of the infection foci contained two or more GFP-positive cells when p35X12 was co-bombarded with piX.erG3(8dm) (Fig. 3J and Table 2). This means that the movement-defective phenotype of the p8-deficient mutant PVX.erG3(8dm) can be restored when an increased amount of p12 is expressed. No such effect was observed for $\mathrm{p} 25$ or CP (Table 2). Overexpression of p12 did not rescue the defect in the p25 or CP gene (Fig. $3 \mathrm{~K}$ and $\mathrm{L}$ and Table 2). Therefore, the leaky phenotype of the p8-deficient mutants can be ascribed as the activity of p12.

\section{p8 functions intracellularly.}

The overall size of infection foci resulting from transcomplementation by $\mathrm{p} 8$ was rather small compared with the other three proteins examined (data not shown), which is consistent with the idea that $\mathrm{p} 8$ functions only in the expressing cells (Lough et al. 2000). To confirm this, we bombarded plasmids piX.erG3(8dm), p35X8, and pBImGFP5ER (Fig. 1). In most of the sites (91\%) where movement defectiveness of PVX.erG3(8dm) was transcomplemented, secondary infected cells were all connected to the bombarded cells (Fig. 4E and F and Table 3). In the rest of the sites, one or a few infected cells were separated from the bombarded cells by two or more cell boundaries (Table 3 ). This level (9\% of the sites) could be explained by the fact that PVX can move at a low level in the absence of p8 (Table 2). Taken together, it was concluded that

Table 2. Phenotype of Potato virus X (PVX) mutants and transcomplementation of their movement defect

\begin{tabular}{|c|c|c|c|c|c|}
\hline \multirow[b]{2}{*}{ PVX mutant ${ }^{\mathrm{a}}$} & \multirow[b]{2}{*}{ Co-expressed protein ${ }^{b}$} & \multicolumn{3}{|c|}{ No. of infected cells in each infection site ${ }^{c}$} & \multirow[b]{2}{*}{ Frequency of cell-to-cell movement $(\%)^{\mathrm{d}}$} \\
\hline & & One & Two & Three or more & \\
\hline PVX.erG3 & - & 0 & 1 & 46 & 100 \\
\hline PVX.erG3(25fs) & - & 62 & 0 & 0 & 0 \\
\hline PVX.erG3(12fs) & - & 58 & 0 & 0 & 0 \\
\hline PVX.erG3(8dm) & - & 101 & 13 & 4 & 14 \\
\hline PVX.erG3(Cd) & - & 84 & 0 & 0 & 0 \\
\hline PVX.erG3(TdCd) & - & 37 & 0 & 0 & 0 \\
\hline PVX.erG3(TdCd) & $\mathrm{p} 25+\mathrm{p} 12+\mathrm{CP}$ & 95 & 31 & 18 & 34 \\
\hline PVX.erG3(TdCd) & $\mathrm{p} 25+\mathrm{p} 12+\mathrm{p} 8+\mathrm{CP}$ & 42 & 19 & 177 & 82 \\
\hline PVX.erG3(8dm) & p25 & 100 & 12 & 2 & 13 \\
\hline PVX.erG3(8dm) & p12 & 55 & 32 & 51 & 60 \\
\hline PVX.erG3(8dm) & p8 & 13 & 22 & 105 & 91 \\
\hline PVX.erG3(8dm) & $\mathrm{CP}$ & 73 & 6 & 1 & 9 \\
\hline PVX.erG3(25fs) & p12 & 46 & 0 & 0 & 0 \\
\hline PVX.erG3(Cd) & p12 & 58 & 0 & 0 & 0 \\
\hline
\end{tabular}

${ }^{a}$ PVX mutants were derived from the corresponding piX series plasmids.

${ }^{\mathrm{b}}$ Each protein was expressed from the co-bombarded corresponding $\mathrm{p} 35 \mathrm{X}$ series plasmid. $\mathrm{CP}=$ coat protein.

${ }^{c}$ Number of the green fluorescent protein (GFP)-positive cells in each infection site was counted 3 days postbombardment and categorized into three types: One $=$ single-cell infection; Two $=$ foci consisting of two adjoining cells; and Three or more $=$ foci containing three or more infected cells

${ }^{\mathrm{d}}$ Frequency of cell-to-cell movement is shown as a percentage of the foci composed of two or more GFP-positive cells in the total number of foci counted. 
p8 functions inside the cells, expressing it as a facilitator for p12, and does not traffic between cells, at least as an active protein.

\section{DISCUSSION}

The ability of plant virus-encoded MPs to increase plasmodesmal permeability is thought to be a requirement for the virus to move through plasmodesmata. Of the PVX-encoded proteins, such an activity was ascribed previously to p25 on the basis of the results obtained from microinjection experiments (Lough et al. 1998). Storms and colleagues (1998), however, pointed out from a comparative study of two microinjection methods that the increase in the plasmodesmal SEL in tobacco tissues expressing a tobamoviral MP or a tospoviral MP might not reflect a genuine biochemical activity of the MP, compelling a reexamination for microinjection-based argumentation. In the present study, we employed an alternative method based on the assumption that the enhanced cell-to-cell trafficking of a soluble GFP expressed in planta reflects the increase in plasmodesmal permeability (Oparka et al. 1999; Satoh et al. 2000; Tamai and Meshi 2001). The results show that p12 has the ability to increase plasmodesmal permeability, at least in the epidermal cells of $N$. benthamiana (Fig. 2B and Table 1). We could not detect such activity for $\mathrm{p} 25$, $\mathrm{p} 8$, or CP (Fig. 2A, C, and D and Table 1).

We also found by transcomplementation experiments that the bombarded p12 gene can function not only in the initially infected (bombarded) cells but also in the surrounding cells, enabling the p12-deficient mutant to spread through two or more cell boundaries (Fig. 4C and Table 3 ). This suggests that p12 trafficked from the bombarded cells to neighboring cells through plasmodesmata, perhaps along with the genomic RNA. These characteristics of p12, i.e., the ability to modulate the plasmodesmal permeability and to promote its own trafficking as well as viral movement in infection sites, are those which are attributable to the MPs.

Previously, Lough and colleagues (2000) employed transcomplementation experiments nearly identical to ours, but they concluded that $\mathrm{p} 12$, like $\mathrm{p} 8$, only functions intracellularly. Although the reason for this discrepancy is unclear, it should be noted that virally expressed visible markers differ in that Lough and coworkers (2000) used the GFP-2A-CP fusion, whereas we used an ER-localized GFP. The GFP-2A-CP fusion is partly self-processed to release a functional $\mathrm{CP}$, and the unprocessed fusion was suggested to interfere with viral cellto-cell movement (Santa Cruz et al. 1996). Taken together, the GFP-2A-CP fusion could somehow be inhibiting the function of one or more TGB-encoded proteins.

Whereas most microinjection studies trace the trafficking of macromolecules within minutes, Angell and colleagues (1996) carefully examined the infection-coupled changes of plasmodesmal SEL at 1 day after injection into trichome cells of Nicotiana clevelandii and showed that, although higher than the noninfected control cells, cell-to-cell trafficking of fluorescently labeled, high-molecular-weight dextran was impaired greatly in the cells infected (co-injected) with a p25-deficient mutant compared with the cells infected with a movementcompetent PVX. Together with the results of the conventional microinjection assays, Angell and colleagues (1996) suggested that p25 is not solely responsible for the infection-coupled increase in plasmodesmal SEL and that other virus-coded protein(s), including p12 and p8, might be involved. In light of our findings, the residual activity Angell and coworkers (1996) observed for the p25-deficient mutant can be ascribed to the activity of p12. It seems plausible that a higher level of plasmodesmal permeability is accompanied with infection of wild-type PVX, considering the distorted appearance of plasmodesmata in infection sites such as the presence of virions (Oparka et al. 1996; Rouleau et al. 1995; Santa Cruz et al. 1998) and the lack of a desmotubule (Santa Cruz et al. 1998).

Transcomplementation experiments indicate that p25 and $\mathrm{CP}$ can function multicellularly in viral cell-to-cell movement (Fig. 4A and D and Table 3). Thus, these proteins are likely to move through plasmodesmata together with the genomic RNA when PVX movement is well conditioned. Although neither p25 nor CP exhibited the plasmodesmal permeability-modulating activity in our assays (Fig. 2A and D and Table 1), it cannot be denied that, during the course of viral cell-to-cell movement, p25 and CP may modify some plasmodesmal function in concert with other viral products.

Yang and colleagues (2000) showed that GFP-p25 (GFP fused to the $\mathrm{N}$ terminus of $\mathrm{p} 25$ ) can move between cells when expressed in the epidermal cells of tobacco by the bombardment-mediated method. Together with the results of microinjection studies (Lough et al. 1998), p25 was suggested to pro-

Table 3. Ability of transiently expressed triple gene block-encoded proteins and the coat protein (CP) to transcomplement the movement-defective phenotype of Potato virus $X(\mathrm{PVX})$ mutants

\begin{tabular}{|c|c|c|c|}
\hline \multirow[b]{2}{*}{ Bombarded plasmids $^{b}$} & \multicolumn{2}{|c|}{ No. of cell boundaries ${ }^{a}$} & \multirow[b]{2}{*}{ Total infection sites ${ }^{\mathrm{a}}$} \\
\hline & One $^{\mathrm{c}}$ & Two or more ${ }^{c}$ & \\
\hline piX.erG3(25fs) + p35X25 + pBImGFP5ER & $16(17)$ & $88(83)$ & $104(100)$ \\
\hline piX.erG3(12fs) + p35X12 + pBImGFP5ER & $22(24)$ & $68(76)$ & $90(100)$ \\
\hline piX.erG3 $(8 \mathrm{dm})+$ p35X8 + pBImGFP5ER & $64(91)$ & $6(9)$ & $70(100)$ \\
\hline piX.erG3 $(\mathrm{Cd})+\mathrm{p} 35 \mathrm{XCP}+\mathrm{pBImGFP5ER}$ & $9(17)$ & $44(83)$ & $53(100)$ \\
\hline \multicolumn{4}{|c|}{$\begin{array}{l}\text { a Infection foci each having two or more infected cells and one mGFP5ER-positive cell were counted. Single-cell infections were not counted because } \\
\text { they could not be discriminated from noninfected mGFP5ER-positive cells, which were visualized by UV and blue-light irradiation. Typical patterns } \\
\text { observed for the respective combinations are shown in Figure } 4 \text {. }\end{array}$} \\
\hline \multicolumn{4}{|c|}{$\begin{array}{l}\text { The structures of the bombarded plasmids are shown in Figure } 1 \text {. The piX series plasmids produced replication-competent PVX with a mutation or mu- } \\
\text { tations in one of the p25, p12, p8, and CP open reading frames. p35X series plasmids expressed p25, p12, p8, and CP under the control of the } 35 \mathrm{~S} \text { pro- } \\
\text { moter. pBImGFP5ER expressing UV-excitable and nontrafficking mGFP5ER was included to identify the bombarded cells. }\end{array}$} \\
\hline \multicolumn{4}{|c|}{$\begin{array}{l}{ }^{\mathrm{c}} \text { Data are presented as the number of infection sites in which the movement-defective virus spread across the indicated number of cell boundaries: one, } \\
\text { all the secondary infected cells were connected to the bombarded cell; two or more, at least one of the secondary infected cells was not connected di- } \\
\text { rectly to the bombarded cell. Frequency }(\%) \text { is shown in parentheses. }\end{array}$} \\
\hline
\end{tabular}


mote its own cell-to-cell trafficking, without the help of other viral factors. In this context, our observation that $\mathrm{p} 25 \mathrm{did}$ not enhance the cell-to-cell trafficking of co-expressed G3GFP means that p25 moved through plasmodesmata by itself, without modulating their permeability. When considering the following observations, however, additional experiments are inevitable to elucidate the true nature of nontagged, wild-type p25. p25-GFP fusions (GFP fused to the $C$ terminus of p25) that have been examined so far are all form aggregates (Malcuit et al. 1999; Morozov et al. 1999; Yang et al. 2000), which is in striking contrast with the GFP-p25 designed by Yang and colleagues (2000) that was dispersed throughout the cytoplasm and nucleus. p25-GFP did not move between cells

\section{Blue light}

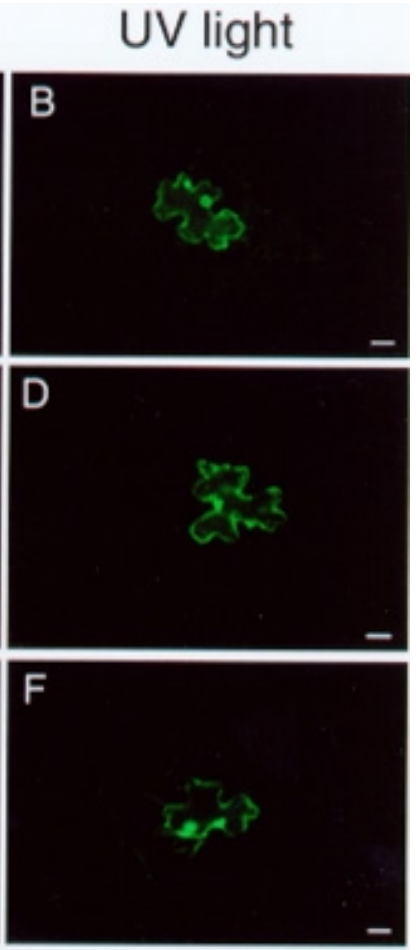

$\mathrm{H}$



Fig. 4. Activity of the triple gene block-encoded proteins and the coat protein transcomplementation of the movement-defective PVX mutants. A and B, Co-bombardment of piX.erG3(25fs), p35X25, and pBImGFP5ER encoding PVX.erG3(25fs), p25, and mGFP5ER, respectively. C and D, Co-bombardment of piX.erG3(12fs), p35X12, and pBImGFP5ER encoding PVX.erG3(12fs), p12, and mGFP5ER, respectively. $\mathbf{E}$ and F, Co-bombardment of piX.erG3(8dm), p35X8, and pBImGFP5ER encoding PVX.erG3(8dm), p8, and mGFP5ER, respectively. G and H, Co-bombardment of piX.erG3(Cd), p35XCP, and pBImGFP5ER, encoding PVX.erG3(Cd), CP, and mGFP5ER, respectively. Images were taken under blue-light irradiation $(\mathbf{A}, \mathbf{C}, \mathbf{E}$, and $\mathbf{G})$ and under UV irradiation (B, D, F, and $\mathbf{H})$. The initially infected (bombarded) cells identified in $\mathbf{B}, \mathbf{D}, \mathbf{F}$, and $\mathbf{H}$ are traced in red in $\mathbf{A}, \mathbf{C}, \mathbf{E}$, and $\mathbf{G}$, respectively. Arrows in A, C, and $\mathbf{G}$ indicate cells not connected directly with the initially infected cells. Images were taken 3 days postbombardment. Bars = $25 \mu \mathrm{m}$. in tobacco (Yang et al. 2000), potato (Malcuit et al. 1999), or $N$. benthamiana (our unpublished observation). Nevertheless, p25-GFP was able to transcomplement a p25-deficient mutant, at least in N. benthamiana (Morozov et al. 1999; our unpublished observation). Thus, it appears that p25 and its derivatives can fulfill their functions in viral cell-to-cell movement, irrespective of whether they have infection-independent cell-to-cell trafficking activity.

The p8-deficient mutants exhibit a leaky phenotype, indicating that $\mathrm{p} 8$ is not absolutely required for cell-to-cell movement but is necessary for efficient movement (Fig. 3E, F, and $\mathrm{H}$ and Table 2). The expression of $\mathrm{p} 8$ transcomplemented the p8-deficient mutants, enabling them to spread to immediately adjacent cells (Fig. 4E and Table 3). Therefore, p8 appears to only function intracellularly and not beyond the plasmodesmata of the cells expressing it. Interestingly, the impaired movement of the p8 mutant was restored considerably by overexpression of p12 (Fig. 3J and Table 2). Thus, p12 can fulfill its function in the absence of $\mathrm{p} 8$, albeit quite inefficiently, and p8 probably functions as an intracellular facilitator for $\mathrm{p} 12$.

PVX p8 has been localized by immunohistochemistry in the cell periphery and the cell wall (Hefferon et al. 1997). Solovyev and colleagues (2000) showed that when TGBp3 of Poa semilatent virus (PSLV, a hordeivirus) or PVX was coexpressed in $N$. benthamiana with the TGBp2-GFP fusion of PSLV or PVX, either TGBp3 could target both TGBp2-GFP fusions from membrane-related structures in the cortical regions to the peripheral compartment. On the basis of these observations, it is inferred that $\mathrm{p} 8$ facilitates the intracellular trafficking of p12. In the absence of p8, the intracellular distribution of $\mathrm{p} 12$ might be more random.

The form in which PVX moves from cell to cell is thought to be a viral ribonucleoprotein (vRNP) that is either like a virion (Oparka et al. 1996; Santa Cruz et al. 1998) or distinct from it (Lough et al. 1998; Lough et al. 2000). The newly synthesized genomic RNA would be assembled into the vRNP, in or close to the cytoplasmic laminate inclusions where p25 is predominantly found (Davies et al. 1993) and RNA is actively synthesized (Kraev and Kushnirenko 1981). The RNA binding activity of p25 and CP likely is involved in the vRNP assembly. The finding that $\mathrm{p} 25, \mathrm{p} 12$, and $\mathrm{CP}$ can function multicellularly suggests that these three proteins traffic through plasmodesmata together with the genomic RNA. It is therefore possible that $\mathrm{p} 12, \mathrm{p} 25$, and $\mathrm{CP}$ may be associated with the maturated vRNP. The function of $\mathrm{p} 8$ could be to facilitate intracellular transport of vRNP from the replication or vRNP assembly site to the peripheral regions of the infected cells through direct or indirect interaction with p12. It also is possible to speculate that expression of $\mathrm{p} 8$ may cause an increase in the intracellular level of active p12, e.g., by facilitating a conformational change of $\mathrm{p} 12$ or by somehow enhancing its expression.

p12 is expected to play a pivotal role in opening the plasmodesmal gate. During trafficking through plasmodesmata, p25 and CP also could interact, even if transiently, with some plasmodesmal factors. Considering the requirement of $\mathrm{p} 8$ in every infected cell for efficient transport, at least some of the initially transported vRNP must undergo disassembly, translation, and replication to finally produce a sufficient amount of p8. p25 may be involved in disassembly and translation in the 
secondary infected cells, as suggested by Atabekov and colleagues (2000). After the amount of $\mathrm{p} 8$ in the secondary infected cells is sufficient, some transported vRNP might be moved directly to the tertiary cells, without entering the replication cycle. It also is plausible that $\mathrm{p} 25, \mathrm{p} 12$, and CP transported into the secondary infected cells can be reutilized there in order to move the newly replicated genomic RNA further away.

TGBp2 and TGBp3, including p12 and p8, have properties of membrane proteins (Donald et al. 1993; Hefferon et al. 1997; Morozov et al. 1990; Morozov et al. 1991; NiesbachKlösgen et al. 1990). If $\mathrm{p} 12$ and $\mathrm{p} 8$ are associated with (or integrated into) membranes throughout the movement process, the intracellular and intercellular trafficking of vRNP may be a membrane-mediated process. In conclusion, the findings in this work stress the importance of TGBp2 in the cell-to-cell movement of TGB-containing plant viruses. In support of this conclusion, TGBp2 is most conserved among TGB-encoded proteins, in terms of size and amino acid sequence.

\section{MATERIALS AND METHODS}

\section{Plasmids for transient expression}

of TGB-encoded proteins and CP of PVX.

A DNA fragment encoding a TGB-encoded protein or CP of PVX (O strain) was amplified by polymerase chain reaction (PCR) from cDNA clone pPVX-UK3/OIII, from which an infectious RNA can be transcribed. PCR primers used were TGB-fw， 5'-CCTTCTAGATTTGAATAAGATGGATATT-3'; TGB-rev, 5'-ACAGAGCTCGAGTATCAATGGAAACT-3'; 8K-fw, 5'-TGCTCTAGACTTTACTGATCTATGG-3'; CP-fw, 5'-TGATCTAGAAAAGATGTCAGCACCAGC-3'; and CPrev, 5'-ACTGAGCTCTGGGGTAGGCGTCGGTT-3'. Underlined bases indicate artificially introduced $\mathrm{XbaI}$ (TCTAGA) or SacI (GAGCTC) sites, the initiation codons (ATG), and the termination codon (complementary to TGA). The TGB-containing PCR fragment was amplified with primers TGB-fw and TGB-rev and cut with $\mathrm{XbaI}$ to yield a $0.78-\mathrm{kbp}$ fragment containing the p25 ORF. This fragment was blunt ended, then inserted between the blunt-ended $X b a \mathrm{I}$ and $S a c \mathrm{I}$ sites of pBI221 (Clontech, Palo Alto, CA, U.S.A.) to create p35X25 (Fig. 1A). The same PCR fragment amplified with TGB-fw and TGB-rev was cut with $N d e I$ and $S c a$ I to yield the $0.35-\mathrm{kbp}$ fragment only with the p12 ORF. This fragment was blunted and inserted between the SmaI and SacI (blunted) sites of pBI221 to create $\mathrm{p} 35 \mathrm{X} 12$. The $\mathrm{p} 8$ and CP ORFs were amplified with primers $8 \mathrm{~K}-\mathrm{fw}$ and TGB-rev and with $\mathrm{CP}-\mathrm{fw}$ and $\mathrm{CP}-$ rev, respectively. These fragments were then cut with $\mathrm{XbaI}$ and $\mathrm{SacI}$ and inserted into the corresponding sites of $\mathrm{pBI} 221$ to create $\mathrm{p} 35 \mathrm{X} 8$ and $\mathrm{p} 35 \mathrm{XCP}$ (Fig. 1A). All PCR-amplified regions and ligation junctions were confirmed by sequencing. In each case, the first ATG from the transcription initiation site corresponded to the authentic initiation codon. Note that the amino acid sequence of p12 is identical between the UK3 and $\mathrm{O}$ strains. p35LM and p35YM, from which the ToMV (L strain) 30K MP and CMV (Y strain) 3a MP are expressed, are described elsewhere (Tamai and Meshi 2001).

\section{Infectious PVX plasmids expressing erG3GFP.}

The coding sequence for erG3GFP was prepared from pBIerG3 (Tamai and Meshi 2001) as a XbaI-blunted SacI fragment. This fragment was ligated with the SalI (blunted)XhoI and NheI-XhoI fragments of pPVX201, a PVX vector derived from the UK3 strain (Baulcombe et al. 1995), to generate piX.erG3 (Fig. 1). The frame-shift mutations were introduced at the ApaI (nucleotide 4,945) or XbaI (nucleotide 5,249 ) site of piX.erG3 by Klenow treatment to create piX.erG(25fs) and piX.erG3(12fs). The sequence from nucleotides 5,667 to 6,302 in the CP ORF was removed from piX.erG3 with NheI and XhoI to generate piX.erG3(Cd). piX.erG3 $(8 \mathrm{dm})$ was generated via intermediates. First, a fragment encoding the C-terminal portion of $\mathrm{p} 8$ (nucleotide 5,539 to the end of the p8 ORF) and erG3GFP was PCR amplified from piX.erG3 with primers 5'-TTACCCGGGAATCAATCACAGTGTTG-3' (SmaI site is underlined) and 5'AAGACTAGTTTATTTGTATAGTTCATCCATGCC-3' and digested with $S m a \mathrm{I}$ and $N s p \mathrm{~V}$. The resulting fragment was inserted between the ApaI and NspV sites of piX.erG3 together with the ApaI-ScaI fragment of pPVX-UK3/OIII. To remove the $\mathrm{O}$ strain-derived sequence from the resulting plasmid, the sequence from the $X b a \mathrm{I}$ site (nucleotide 5,254 in the p12 ORF) to the $N s p \mathrm{~V}$ site in the erG3GFP ORF was replaced with the corresponding fragment of piX.erG3. Finally, the initiation codon of the disrupted p8 ORF was mutated from ATG to ACG by a PCR-based mutagenesis procedure to make piX.erG3(8dm). The plasmid piX.erG3(TdCd) was constructed by ligating the ApaI (blunted) $-N s p \mathrm{~V}$ fragment of piX.erG3(Cd) and the $\mathrm{XbaI}$ (blunted) $-N s p \mathrm{~V}$ fragment of piX.erG3(8dm).

\section{Microprojectile bombardment.}

Bombardment was performed with PDS1000 (Bio-Rad Laboratories, Hercules, CA, U.S.A.), as described elsewhere (Tamai and Meshi 2001). Briefly, mature leaves (8 to $10 \mathrm{~cm}$ in length) of $N$. benthamiana (6 to 8 weeks old) were cut, placed on a Murashige-Skoog agar plate at a target distance of $6 \mathrm{~cm}$, and bombarded with a rupture disk of $1,100 \mathrm{lb} / \mathrm{in}^{2}$. Three milligrams of $1-\mu \mathrm{m}$ gold particles were coated with $5 \mu \mathrm{g}$ of plasmid DNA. In co-bombardment experiments, equal amounts of the respective plasmids (totaling $5 \mu \mathrm{g}$ ) were mixed before coating the gold particles. Bombarded leaves were incubated at $26^{\circ} \mathrm{C}$ in the dark for 1 to 3 days. GFP signals were observed under an Axioskop epifluorescent microscope (Carl Zeiss, Esslingen, Germany) equipped with the C5810 color-chilled 3CCD camera (Hamamatsu Photonics, Hamamatsu, Japan). Filter sets 41014 and 31022 (Chroma Technologies, Brattleboro, VT, U.S.A.) were used to observe GFP variants under blue-light and UV irradiation, respectively.

\section{ACKNOWLEDGMENTS}

We are deeply indebted to D. Baulcombe for his supply of the infectious PVX clone pPVX201. We also thank J. Haseloff for a plasmid encoding mGFP5ER, and $\mathrm{H}$. Takahashi for a PVX cDNA clone. This work was supported in part by a grant-in-aid for scientific research from the Ministry of Education, Science, Sports and Culture of Japan.

\section{LITERATURE CITED}

Angell, S. M., Davies, C., and Baulcombe, D. C. 1996. Cell-to-cell movement of potato virus $\mathrm{X}$ is associated with a change in the sizeexclusion limit of plasmodesmata in trichome cells of Nicotiana clevelandii. Virology 216:197-201. 
Ares, X., Calamante, G., Cabral, S., Lodge, J., Hemenway, P., Beachy, R. N., and Mentaberry, A. 1998. Transgenic plants expressing potato virus $\mathrm{X}$ ORF2 protein (p24) are resistant to tobacco mosaic virus and Ob tobamoviruses. J. Virol. 72:731-738.

Atabekov, J. G., and Taliansky, M. E. 1990. Expression of a plant viruscoded transport function by different viral genomes. Adv. Virus Res. 38:201-248.

Atabekov, J. G., Rodionova, N. P., Karpova, O. V., Kozlovsky, S. V., and Poljakov, V. Y. 2000. The movement protein-triggered in situ conversion of potato virus $\mathrm{X}$ virion RNA from a nontranslatable into a translatable form. Virology 271:259-263.

Baulcombe, D. C., Chapman, S., and Santa Cruz, S. 1995. Jellyfish green fluorescent protein as a reporter for virus infections. Plant J. 7:1045-1053

Beck, D. L., Guilford, P. J., Voot, D. M., Andersen, M. T., and Forster, R. L. S. 1991. Triple gene block proteins of white clover mosaic potexvirus are required for transport. Virology 183:659-702.

Bleykasten, C., Gilmer, D., Guilley, H., Richards, K. E., and Jonard, G. 1996. Beet necrotic yellow vein virus $42 \mathrm{kDa}$ triple gene block protein binds nucleic acids in vitro. J. Gen. Virol. 77:889-897.

Carrington, J. C., Kasschau, K. D., Mahajan, S. K., and Schaad, M. C. 1996. Cell-to-cell and long-distance transport of viruses in plants. Plant Cell 8:1669-1681.

Chang, B. Y., Lin, N. S., Liou, D. Y., Chen, J. P., Liou, G. G., and Hsu, Y. H. 1997. Subcellular localization of the $28 \mathrm{kDa}$ protein of the triple-gene-block of bamboo mosaic potexvirus. J. Gen. Virol. 78:11751179.

Chapman, S., Hills, G., Watts, J., and Baulcombe, D. 1992. Mutational analysis of the coat protein gene of potato virus X: Effects on virion morphology and viral pathogenicity. Virology 191:223-230.

Davies, C., Hills, G., and Baulcombe, D. C. 1993. Sub-cellular localization of the $25-\mathrm{kDa}$ protein encoded in the triple gene block of potato virus X. Virology 197:166-175.

Deom, C. M., Lapidot, M., and Beachy, R. N. 1992. Plant virus movement proteins. Cell 69:221-224.

Ding, B., Li, Q., Nguyen, L., Palukaitis, P., and Lucas, W. J. 1995. Cucumber mosaic virus 3 a protein potentiates cell-to-cell trafficking of CMV RNA in tobacco plants. Virology 207:345-353.

Donald, R. G. K., Shou, H., and Jackson, A. O. 1993. Serological analysis of barley stripe mosaic virus-encoded proteins in infected barley. Virology 195:659-668.

Donald, R. G. K., Lawrence, D. M., and Jackson, A. O. 1997. The barley stripe mosaic virus 58-kilodalton $\beta \mathrm{b}$ protein is a multifunctional RNA binding protein. J. Virol. 71:1538-1546.

Forster, R. L. S., Beck, D. L., Guilford, P. J., Voot, D. M., Van Dolleweerd, C. J., and Andersen, M. T. 1992. The coat protein of white clover mosaic potexvirus has a role in facilitating cell-to-cell transport in plants. Virology 191:480-484

Gilmer, D., Bouzoubaa, S., Hehn, A., Guilley, H., Richards, K., and Jonard, G. 1992. Efficient cell-to-cell movement of beet necrotic yellow vein virus requires $3^{\prime}$ proximal genes located on RNA 2. Virology 189:40-47.

Gorbalenya, A. E., and Koonin, E. V. 1989. Viral proteins containing the purine NTP-binding sequence pattern. Nucleic Acids Res. 17:84138440.

Haseloff, J., and Siemering, K. R. 1998. The uses of green fluorescent protein in plants. Pages 191-220 in: Green Fluorescent Protein: Properties, Applications, and Protocols. M. Chalfie and S. Kain, eds. Wiley-Liss, New York.

Hefferon, K. L., Doyle, S., and AbouHaidar, M. G. 1997. Immunological detection of the $8 \mathrm{~K}$ protein of potato virus $\mathrm{X}(\mathrm{PVX})$ in cell walls of PVX-infected tobacco and transgenic potato. Arch. Viol. 142:425433.

Herzog, E., Hemmer, O., Hauser, S., Meyer, G., Bouzoubaa, S., and Fritsch, C. 1998. Identification of genes involved in replication and movement of peanut clump virus. Virology 248:312-322.

Kalinina, N. O., Fedorkin, O. N., Samuilova, O. V., Maiss, E., Korpela, T., Morozov, S. Y., and Atabekov, J. G. 1996. Expression and biochemical analyses of the recombinant potato virus $\mathrm{X} 25 \mathrm{~K}$ movement protein. FEBS Lett. 397:75-78.

Kawakami, S., and Watanabe, Y. 1997. Use of green fluorescent protein as a molecular marker tag of protein movement in vivo. Plant Biotech. 14:127-130.

Kraev, V. G., and Kushnirenko, O. A. 1981. Localization of potato X- virus RNA synthesis in cells of Datura stramonium L. Mikrobiol. Zh. (Kiev) 43:619-626.

Lazarowitz, S. G., and Beachy, R. N. 1999. Viral movement proteins as probes for intracellular and intercellular trafficking in plants. Plant Cell 11:535-548.

Liou, D. Y., Hsu, Y. H., Wung, C. H., Wang, W. H., Lin, N. S., and Chang, B. Y. 2000. Functional analyses and identification of two arginine residues essential to the ATP-utilizing activity of the triple gene block protein 1 of bamboo mosaic potexvirus. Virology 277:336-344.

Lough, T. J., Shash, K., Xoconostle-Cázares, B., Hofstra, K. R., Beck, D. L., Balmori, E., Forster, R. L. S., and Lucas, W. J. 1998. Molecular dissection of the mechanism by which potexvirus triple gene block proteins mediate cell-to-cell transport of infectious RNA. Mol. PlantMicrobe Interact. 11:801-814.

Lough, T. J., Netzler, N. E., Emerson, S. J., Sutherland, P., Carr, F., Beck, D. L., Lucas, W. J., and Forster, R. L. S. 2000. Cell-to-cell movement of potexviruses: evidence for a ribonucleoprotein complex involving the coat protein and first triple gene block protein. Mol. Plant-Microbe Interact. 13:962-974.

Lucas, W. J., Ding, B., and Van der Schoot, C. 1993. Plasmodesmata and the supracellular nature of plants. New Phytol. 125:435-476.

Malcuit, I., Marano, M. R., Kavanagh, T. A., De Jong, W., Forsyth, A., and Baulcombe, D. C. 1999. The 25-kDa movement protein of PVX elicits $\mathrm{Nb}$-mediated hypersensitive cell death in potato. Mol. PlantMicrobe Interact. 12:536-543.

Maule, A. J. 1991. Virus movement in infected plants. Crit. Rev. Plant Sci. 9:457-473.

Morozov, S. Y., Dolja, V. V., and Atabekov, J. G. 1989. Probable reassortment of genomic elements among elongated RNA-containing plant viruses. J. Mol. Evol. 29:52-62.

Morozov, S. Y., Miroshnichenko, N. A., Zelenina, D. A., Fedorkin, O. N., Solovyev, A. G., Lukasheva, L. I., and Atabekov, J. G. 1990. Expression of RNA transcripts of potato virus X full-length and subgenomic cDNAs. Biochimie 72:677-684.

Morozov, S. Y., Miroshnichenko, N. A., Solovyev, A. G., Zelenina, D. A., Fedorkin, O. N., Lukasheva, L. I., Grachev, S. A., and Chernov, B. K. 1991. In vitro membrane binding of the translation products of the carlavirus 7-kDa protein genes. Virology 183:782-785.

Morozov, S. Y., Fedorkin, O. N., Jüttner, G., Schiemann, J., Baulcombe, D. C., and Atabekov, J. G. 1997. Complementation of a potato virus X mutant mediated by bombardment of plant tissues with cloned viral movement protein genes. J. Gen. Virol. 78:2077-2083.

Morozov, S. Y., Solovyev, A. G., Kalinina, N. O., Fedorkin, O. N., Samuilova, O. V., Schiemann, J., and Atabekov, J. G. 1999. Evidence for two nonoverlapping functional domains in the potato virus $\mathrm{X} 25 \mathrm{~K}$ movement protein. Virology 260:55-63.

Niesbach-Klösgen, U., Guilley, H., Jonard, G., and Richards, K. 1990 Immunodetection in vivo of beet necrotic yellow vein virus-encoded proteins. Virology 178:52-61.

Oparka, K. J., Roberts, A. G., Roberts, I. M., Prior, D. A. M., and Santa Cruz, S. 1996. Viral coat protein is targeted to, but does not gate, plasmodesmata during cell-to-cell movement of potato virus X. Plant $\mathrm{J}$. 10:805-813.

Oparka, K. J., Roberts, A. G., Boevink, P., Santa Cruz, S., Roberts, I., Pradel, K. S., Imlau, A., Kotlizky, G., Sauer, N., and Epel, B. 1999. Simple, but not branched, plasmodesmata allow the nonspecific trafficking of proteins in developing tobacco leaves. Cell 97:743-754.

Overall, R. L., and Blackman, L. M. 1996. A model of the macromolecular structure of plasmodesmata. Trends Plant Sci. 1:307-311.

Petty, I. T. D., and Jackson, A. O. 1990. Mutational analysis of barley stripe mosaic virus RNA $\beta$. Virology 179:712-718.

Rouleau, M., Smith, R. J., Bancroft, J. B., and Mackie, G. A. 1994. Purification, properties, and subcellular localization of foxtail mosaic potexvirus 26-kDa protein. Virology 204:254-265.

Rouleau, M., Smith, R. J., Bancroft, J. B., and Mackie, G. A. 1995. Subcellular immunolocalization of the coat protein of two potexviruses in infected Chenopodium quinoa. Virology 214:314-318.

Santa Cruz, S., Chapman, S., Roberts, A. G., Roberts, I. M., Prior, D. A M., and Oparka, K. J. 1996. Assembly and movement of a plant virus carrying a green fluorescent protein overcoat. Proc. Natl. Acad. Sci. USA 93:6286-6290.

Santa Cruz, S., Roberts, A. G., Prior, D. A. M., Chapman, S., and Oparka, K. J. 1998. Cell-to-cell and phloem-mediated transport of potato virus X: The role of virions. Plant Cell 10:495-510. 
Satoh, H., Matsuda, H., Kawamura, T., Isogai, M., Yoshikawa, N., and Takahashi, T. 2000. Intracellular distribution, cell-to-cell trafficking and tubule-inducing activity of the $50 \mathrm{kDa}$ movement protein of $\mathrm{Ap}$ ple chlorotic leaf spot virus fused to green fluorescent protein. J. Gen. Virol. 81:2085-2093.

Solovyev, A. G., Savenkov, E. I., Agranovsky, A. A., and Morozov, S. Y 1996. Comparisons of the genomic cis-elements and coding regions in RNA $\beta$ components of the hordeiviruses barley stripe mosaic virus, lychnis ringspot virus, and poa semilatent virus. Virology 219:9-18.

Solovyev, A. G., Stroganova, T. A., Zamyatnin, A. A., Jr., Fedorkin, O. N., Schiemann, J., and Morozov, S. Y. 2000. Subcellular sorting of small membrane-associated triple gene block proteins: TGBp3assisted targeting of TGBp2. Virology 269:113-127.

Spillane, C., Verchot, J., Kavanagh, T. A., and Baulcombe, D. C. 1997. Concurrent suppression of virus replication and rescue of movementdefective virus in transgenic plants expressing the coat protein of potato virus X. Virology 236:76-84.

Storms, M. M. H., Van der Schoot, C., Prins, M., Kormelink, R., Van Lent, J. W. M., and Goldbach, R. W. 1998. A comparison of two methods of microinjection for assessing altered plasmodesmal gating in tissues expressing viral movement proteins. Plant J. 13:131-140.

Tamai, A., and Meshi, T. 2001. Tobamoviral movement protein transiently expressed in a single epidermal cell functions beyond multiple plasmodesmata and spreads multicellularly in an infection-coupled manner. Mol. Plant-Microbe Interact. 14:126-134.

Van Bel, A. J. E., and Van Kesteren, W. J. P. 1999. Plasmodesmata:
Structure, Function, Role in Cell Communication. Springer-Verlag, Berlin.

Vaquero, C., Turner, A. P., Demangeat, G., Sanz, A., Serra, M. T., Roberts, K., and García-Luque, I. 1994. The 3a protein from cucumber mosaic virus increases the gating capacity of plasmodesmata in transgenic tobacco plants. J. Gen. Virol. 75:3193-3197.

Verchot, J., Angell, S. M., and Baulcombe, D. C. 1998. In vivo translation of the triple gene block of potato virus $\mathrm{X}$ requires two subgenomic mRNAs. J. Virol. 72:8316-8320.

Waigmann, E., Lucas, W. J., Citovsky, V., and Zambryski, P. 1994. Direct functional assay for tobacco mosaic virus cell-to-cell movement protein and identification of a domain involved in increasing plasmodesmal permeability. Proc. Natl. Acad. Sci. USA 91:1433-1437.

Wolf, S., Deom, C. M., Beachy, R. N., and Lucas, W. J. 1989. Movement protein of tobacco mosaic virus modifies plasmodesmatal size exclusion limit. Science 246:377-379.

Wong, S. M., Lee, K. C., Yu, H. H., and Leong, W. F. 1998. Phylogenetic analysis of triple gene block viruses based on the TGB 1 homolog gene indicates a convergent evolution. Virus Genes 16:295-302.

Wung, C. H., Hsu, Y. H., Liou, D. Y., Huang, W. C., Lin, N. S., and Chang, B. Y. 1999. Identification of the RNA-binding sites of the triple gene block protein 1 of bamboo mosaic potexvirus. J. Gen. Virol. 80:1119-1126.

Yang, Y., Ding, B., Baulcombe, D. C., and Verchot, J. 2000. Cell-to-cell movement of the $25 \mathrm{~K}$ protein of Potato virus $X$ is regulated by three other viral proteins. Mol. Plant-Microbe Interact. 13:599-605. 